Proyecciones

Vol. 14, $\mathrm{N}^{o}$, pp. 115-132, December 1995

Universidad Católica del Norte

Antofagasta - Chile

\title{
ISOCHRONOUS OSCILLATIONS FOR CUBIC SYSTEMS
}

\author{
VICTOR GUINEZ* , \\ Universidad de Santiago de Chile, Santiago - Chile \\ EDUARDO SÁEZ and JAIME FIGUEROA \\ Universidad Técnica Federico Santa María, Valparaíso - Chile
}

\begin{abstract}
Planar vector fields

$$
\begin{aligned}
& \dot{x}=-y+p_{3}(x, y) \\
& \dot{y}=x+q_{3}(x, y) .
\end{aligned}
$$

with a center non necessarily at the origin are considered, where $p_{3}$ and $q_{3}$ are homogeneous polynomials of degree 3 . We are concerned with the behavior of the periods of the periodic solutions near the center, and in determining when the center is isochronous, i.e., when all periodic solutions have the same period. It is proved that, modulus a linear change of coordinates, there are only four systems which have an isochronous center. Each of them has an isochronous center at the origin, and its other centers are also isochronous but not necessarily with the same period. Also the maximal weakness of the non isochronous centers, is obtained.
\end{abstract}

* Supported in part by Fondecyt Grant No.1930881

'Partially supported by Fondecyt Grant No. 1931140 and USM No. 941205 


\section{Introduction.}

In this article we consider symmetric cubic plane vector fields

$$
\begin{aligned}
& \dot{x}=-y+\sum_{i+j=3} a_{i j} x^{i} y^{j}=-y+p_{3}(x, y) \\
& \dot{y}=x+\sum_{i+j=3} b_{i j} x^{i} y^{j}=x+q_{3}(x, y) .
\end{aligned}
$$

which have a center. The question of interest is the behavior of the periods of the periodic solutions near the center, and in determining when the center is isochronous, i.e., when all periodic solutions have the same period. The period function, i.e. the function which associates to any periodic trajectory near a center its minimum period, can be obtained in the following way. Let $(x, y) \rightarrow Z(x, y)$ be a plane analytic vector field with a center at the origin which verifies $D Z(0,0)(x, y)=(-y, x)$. We will call such centers nondegenerated centers. We choose a small open interval $\Sigma$ on the $x$-axis containing the origin such that any orbit passing through a nonzero point of $\Sigma$ is periodic and includes the origin in its interior. For a point $\xi \in \Sigma$, we define $P(\xi)$ to be the minimum period of the periodic trajectory through $\xi$. In polar coordinates the vector field can be written in the form

$$
\frac{d r}{d \theta}=\frac{R(r, \theta)}{1+A(r, \theta)}
$$

and the period function is

$$
P(\xi)=\int_{0}^{2 \pi} \frac{d \theta}{1+A(r(\theta, \xi), \theta)},
$$

where $r=r(\theta, \xi)$ is the solution of equation (1.2) with $r(0, \xi)=\xi$.

The standing hypothesis assures us that the period function will be analytic. Since the first non vanishing derivative of $P$ at 0 must be even, we have

$$
P(\xi)=2 \pi+\sum_{j=2}^{\infty} p_{j} \xi^{j} .
$$

On the other hand, it is well known ([7]) that there is an analytic coordinate transformation $(x, y)=\phi(u, v)$ which transforms our planar analytic system of differential equations with a nondegenerated center at the origin $(\dot{x}, \dot{y})=Z(x, y)$ to the normal form

$$
\dot{u}=-f\left(u^{2}+v^{2}\right) v, \quad \dot{v}=f\left(u^{2}+v^{2}\right) u .
$$


Once this is done, a further change to polar coordinates gives

$$
\dot{r}=0, \quad \dot{\theta}=f\left(r^{2}\right),
$$

and it follows that the period function for (1.5) is given by

$$
\tilde{P}(\zeta)=\frac{2 \pi}{f\left(\zeta^{2}\right)}
$$

for all $\zeta$ in a small open interval $\tilde{\Sigma}$ on the $u$-axis containing the origin.

In particular, a plane analytic vector field with a nondegenerated center at the origin can be transformed to the system $\dot{u}=-v, \dot{v}=u$ with an analytic change of coordinates if and only if the center is isochronous.

If the Taylor expansion for $f\left(\zeta^{2}\right)$ at the origin is

$$
f\left(\zeta^{2}\right)=1+b_{3} \zeta^{2}+b_{5} \zeta^{4}+b_{7} \zeta^{6}+\cdots
$$

then period coefficients $\tilde{p}_{j}$ can be obtained from the relation

$$
\frac{2 \pi}{1+b_{3} \zeta^{2}+b_{5} \zeta^{4}+b_{7} \zeta^{6}+\cdots}=2 \pi+\tilde{p}_{2} \zeta^{2}+\tilde{p}_{4} \zeta^{4}+\tilde{p}_{6} \zeta^{6}+\cdots
$$

Of course, $\tilde{P}$ is also the period function for the original vector field $Z(x, y)$ but defined over the curve $\phi(\tilde{\Sigma})$. Therefore, the order and the value of the first non vanishing derivative of $P$ at 0 coincide with the one of $\tilde{P}$.

The coefficients $p_{j}$ in (1.4) are called period coefficients. The center is called a weak center of order $m$ if in (1.4) we have

$$
p_{2}=\ldots=p_{2 m+1}=0 \text { and } p_{2 m+2} \neq 0 .
$$

Otherwise, the center is a weak center of infinite order. Of course, a weak center of infinite order is an isochronal, i.e., all the periodic orbits surrounding the center have the same period.

The corresponding problem for quadratic nondegenerated centers was studied in [2] and [1]. Theorem 4 of [2] gives necessary an sufficient conditions for the coefficients of the field to have an isochronous center at the origin. In the proof of this Theorem the Urabe's criterion ([9]) is used. This criterion can be used for scalar second-order equations of the form

$$
\ddot{u}+g(u)=0
$$


where $g(u)$ is a $C^{1}$-function with $u g(u)>0$ for $u \neq 0$ and $g^{\prime}(0)>0$. It can be described as follows. Define the variable $X$ by

$$
\frac{1}{2} X^{2}=\int_{0}^{u} g(v) d v,
$$

Invert relation (1.7), obtaining $u$ as a function of $X$. Then the solutions of (1.6) will all have the same period if and only if (1.7) is equivalent to

$$
u=X+\text { an even function of } X \text {. }
$$

In [1] the authors compute the period coefficients $p_{2}, p_{4}, p_{6}$ and prove that a weak center of a quadratic system which is not an isochronal has order at most two. They also study the problem of bifurcations of critical points of the period function.

In this paper we study the same problems for symmetric cubic systems. That is, the question is to determine which systems (1.1) have an isochronous center and to determine the maximal weakness when the center is not an isochronal.

The isochronicity of the origin when it is a center for this system was first studied by N. A. Lukashevich ([3]) and I. I. Pleshkan ([5]). Both used the Malkin normal form

$$
\begin{aligned}
& \dot{x}=-y-(\psi+\theta) x^{3}-(\chi-\beta) x^{2} y-(3 \psi-3 \theta-\gamma) x y^{2}-(\mu-\nu) y^{3} \\
& \dot{y}=x+(\mu+\nu) x^{3}+(3 \psi+3 \theta+\alpha) x^{2} y+(\chi+\beta) x y^{2}+(\psi-\theta) y^{3}
\end{aligned}
$$

and the corresponding algebraic characterization of centers ([4]).

In Theorem 7 of [5] Pleshkan proved that the following set of conditions are necessary and sufficient in order that the system (1.8) have an isochronous center at the origin :

PI. $\quad \mu=\chi=\theta=\alpha+\gamma=\beta+3 \nu=\alpha+6 \psi=0$,

PII. $\mu=\chi=\theta=\nu=\alpha+\gamma=\alpha+4 \psi=0$,

PIII. $\chi+3 \mu=\alpha+\gamma=\beta+6 \nu=5 \alpha+24 \psi=4 \psi^{2}+25\left(\nu^{2}-\mu^{2}-\theta^{2}\right)$

$$
=\theta \alpha^{2}-4 \theta \beta^{2}-4 \alpha \beta \mu=0 .
$$

The set of conditions PI and PII contain the ones imposed by Lukashevich in [3]. 
Here we prove that, modulus a linear change of coordinates, the unique symmetric cubic systems which have an isochronous center (non necessarily at the origin) are

$$
\begin{gathered}
\dot{x}=-y\left(1-\frac{3}{2} x^{2}\right) \\
\dot{y}=x\left(1-x^{2}+\frac{9}{2} y^{2}\right) \\
\dot{x}=-y\left(1-9 x^{2}+2 y^{2}\right) \\
\dot{y}=x\left(1+3 y^{2}\right), \\
\dot{x}=-y\left(1-x^{2}\right) \\
\dot{y}=x\left(1+y^{2}\right), \\
\dot{x}=-y\left(1-3 x^{2}+y^{2}\right) \\
\dot{y}=x\left(1-x^{2}+3 y^{2}\right)
\end{gathered}
$$

Each of them verifies Pleshkan's conditions and therefore they have an isochronous center at the origin. We present an independent proof of this isochronicity that also allows us to prove the isochronicity of the centers outside the origin. It is also proved that there are no other symmetric cubic systems with an isochronous center.

We use the same algebraic characterization of centers for systems (1.1) given by Malkin ([4]) but with the simplification introduced by Sibirsky ([8]). This simplification consists in making a rotation to system (1.8) to obtain $a_{21}+b_{12}=0$. He wrote such a system in the form

$$
\begin{gathered}
\dot{\mathrm{x}}=-\mathrm{y}-(\omega+\theta-a) \mathrm{x}^{3}-(\eta-3 \mu) \mathrm{x}^{2} \mathrm{y}-(3 \omega-3 \theta+2 \mathrm{a}-\xi) \mathrm{xy}^{2}-(\mu-\nu) \mathrm{y}^{3} \\
\dot{\mathrm{y}}=\mathrm{x}+(\mu+\nu) \mathrm{x}^{3}+(3 \omega+3 \theta+2 \mathrm{a}) \mathrm{x}^{2} \mathrm{y}+(\eta-3 \mu) \mathrm{xy}^{2}+(\omega-\theta-\mathrm{a}) \mathrm{y}^{3} .
\end{gathered}
$$

The algebraic characterization is the following

Theorem 1. ([4, 8, 6]). System (1.13) has a center at the origin if and only if one of the following conditions is satisfied:

$$
\begin{aligned}
(H) & \xi=a=0 \\
(I I) & \xi=\nu=\theta=0 \\
(I I I) & \xi=\nu=\omega=\eta=4\left(\mu^{2}+\theta^{2}\right)-a^{2}=0 .
\end{aligned}
$$


Condition $(\mathrm{H})$ corresponds to the Hamiltonian systems. Case (II) corresponds to a symmetric system with respect to an axis. Since system (1.13) is symmetric with respect to the origin, such systems necessarily have two symmetry axes. Finally, last case (centers of type III) consists of systems having an invariant algebraic curve of degree 4 and one of degree 6. In [6] the authors give a new proof of the integrability of system (1.13) with a center at the origin using the method of Darboux for all values of the parameters, and give all possible phase portraits of system (1.13) for the nonhamiltonian case.

In this paper we consider system (1.13) with $\lambda=(a, \eta, \theta, \mu, \nu, \xi, \omega) \neq 0$ and we show that these systems under condition $(\mathrm{H})$ have a weak center of order 0 or 1 at the origin (Theorem 10) and under the condition (III) a weak center of order 1 (Theorem 11). Therefore in these cases the origin is never an isochronal. However, in case (II) we have, modulus a linear change of coordinates, exactly four systems which have an isochronous center at the origin (Theorem 2). Two of these systems have centers different from the origin. We prove that they are also isochronous but not necessarily of the same period. Also in case (II), we prove that when the origin is not an isochronal, it is a weak center of order at most three and we give an example where that order is achieved (Corollaries 8 and 9).

Finally we prove that if system (1.1) have an isochronous center outside the origin, then the system can be brought to one of the systems (1.9),(1.12), through a linear change of coordinates.

Our proofs depend on the computation of the first Liapunov quantities $\eta_{2}, \eta_{3}, \eta_{4}$ and of the first period coefficients $p_{2}, p_{4}, p_{6}, p_{8}$. Since these computations involves some complicated calculations with polynomials of higher degree in the coefficients of the vector field, they cannot be completed successfully by hand. Then, as is usual, we developed an algorithm to be implemented on a computer using symbolic manipulations techniques. For this we use the Mathematica computer algebra package on a Hewlett Packard Apollo Series 700 machine in the Federico Santa Maria Technical University (Valparaíso, Chile).

The paper is organized as follows. In the next chapter we present the four systems $1.9,1.10,1.11$ and 1.12 . Chapter 3 is concerned with systems of type (1.13) under condition (II) and Chapter 4 with the ones under conditions (H) and (III). Finally in Chapter 5 it is proved that there are no other systems of form (1.1) with isochronous centers outside the origin. 


\section{Isochronals}

The isochronicity of centers outside the origin for system (1.1) cannot be obtained from Pleshkan's results. Here we present an independent proof of the isochronicity of all centers of the systems $1.9,1.10,1.11$ and 1.12.

The result is the following:

Theorem 2. The origin is an isochronous center for systems (1.9), (1.10), (1.11) and (1.12). Systems (1.10) and (1.11) have no equilibrium points outside the origin. On the other hand, the points $( \pm 1,0)$ are the unique extra equilibrium points for systems (1.9) and (1.12). They are also isochronous centers of period $2 \pi$ for (1.9) and of period $\pi$ for (1.12).

The proof of Theorem 2 will be a consequence of some Propositions which we state below.

Proposition 3. Consider the one-parameter family

$$
\begin{array}{ll}
\dot{x}= & -y\left(1-\frac{3}{2} A x^{2}\right) \\
\dot{y}= & x\left(1-A x^{2}+\frac{9}{2} A y^{2}\right) .
\end{array}
$$

For any value of the parameter $A$ this system has an isochronous center at the origin of period $2 \pi$. If $A \leq 0$ there are no equilibrium points outside the origin. Otherwise we have two equilibrium points outside the origin, which are also isochronous centers and with the same period $2 \pi$.

Proof: By an appropriated change of coordinates, the system can be transformed into a second order differential equation of the form

$$
\ddot{u}+g(u)=0 .
$$

Consider $x=\phi(u)$ with $u=u(t)$, both of class $C^{2}$. Replacing at the first equation of (2.1) we obtain

$$
y=-\frac{\phi^{\prime}(u) \dot{u}}{1-\frac{3}{2} A \phi(u)^{2}} .
$$

where $\phi^{\prime}(u)$ is the derivative of $\phi$ with respect to $u$ and $\dot{u}$ is the derivative of $u$ with respect to the time $t$.

Observe that $1-\frac{3}{2} A \phi(u)^{2}$ is always nonvanishing if $A \leq 0$ and that for positive $A$ it is nonvanishing at a neighborhood of $x=0$ and of $x= \pm \frac{1}{\sqrt{A}}$ which are the first coordinates at the equilibrium points of the system. 
Introducing this in the second equation of (2.1) we obtain

$$
\begin{aligned}
\left(1-\frac{3}{2} A \phi(u)^{2}\right) \phi^{\prime}(u) \ddot{u} & + \\
{\left[\left(1-\frac{3}{2} A \phi(u)^{2}\right) \phi^{\prime \prime}(u)+\frac{15}{2} A\left(\phi^{\prime}(u)^{2} \phi(u)\right] \dot{u}^{2}\right.} & + \\
\phi(u)\left(1-A \phi(u)^{2}\right)\left(1-\frac{3}{2} A \phi(u)^{2}\right)^{2} & =0 .
\end{aligned}
$$

Therefore to obtain an equation of the form (2.2) the map $\phi(u)$ must be solution of

$$
\left(1-\frac{3}{2} A \omega^{2}\right) \omega^{\prime \prime}+\frac{15}{2} A\left(\omega^{\prime}\right)^{2} \omega=0 .
$$

To prove that the origin is an isochronal for system (2.1) we consider this equation with the initial conditions $\omega(0)=0$ and $\omega^{\prime}(0)=1$, which is equivalent to

$$
\omega^{\prime}=\left(1-\frac{3}{2} A \omega^{2}\right)^{\frac{5}{2}}, \quad \omega(0)=0 .
$$

Let $\phi$ be the solution of this last equation. Then $\phi$ verifies the implicit equation

$$
\frac{\phi(u)\left(1-A \phi(u)^{2}\right)}{\left(1-\frac{3}{2} A \phi(u)^{2}\right)^{\frac{3}{2}}}=u .
$$

Introducing this $\phi$ at (2.3), comparing with (2.2) and using relations (2.5) and (2.6) we obtain

$$
g(u)=u
$$

which proves that the origin is isochronous of period $2 \pi$ for system $(2.1)$ for all $A$.

If $A$ is positive, the points $\left( \pm \frac{1}{\sqrt{A}}, 0\right)$ are also centers for our systems. To prove that they are isochronous we solve equation (2.4) with the initial conditions $\omega(0)= \pm \frac{1}{\sqrt{A}}$ and $\omega^{\prime}(0)=1$ which is now equivalent to

$$
\omega^{\prime}=\left[-2\left(1-\frac{3}{2} A \omega^{2}\right)\right]^{\frac{5}{2}}, \quad \omega(0)= \pm \frac{1}{\sqrt{A}} .
$$

Therefore, if $\phi$ is the solution of (2.7), then it must verify the implicit equation

$$
-\frac{\phi(u)\left(1-A \phi(u)^{2}\right)}{2\left[-2\left(1-\frac{3}{2} A \phi(u)^{2}\right)\right]^{\frac{3}{2}}}=u .
$$


Then, as before, introducing this $\phi$ at (2.3), comparing with (2.2) and using relations (2.7) and (2.8) we obtain

$$
g(u)=u
$$

This finishes the proof.

Proposition 4. Consider the one-parameter family of vector fields

$$
\begin{array}{ll}
\dot{x}= & y\left(1-\frac{9}{2} A x^{2}+A y^{2}\right) \\
\dot{y}= & x\left(1+\frac{3}{2} A y^{2}\right) .
\end{array}
$$

For any value of the parameter $A$ this system has an isochronous center at the origin of period $2 \pi$. If $A \geq 0$ there are no equilibrium points other than the origin. Otherwise we have two equilibrium points outside the origin, which are also isochronous centers and with the same period $2 \pi$.

Proof: The transformation $\phi(x, y)=(y,-x)$ turns system (2.9) into system (2.1) with $-A$ instead of $A$. Then the proof follows from Proposition 3.

Proposition 5. System (1.11) has an isochronous center of period $2 \pi$ at the origin.

Proof: In polar coordinates

$$
x=r \cos \theta, \quad y=r \sin \theta
$$

the system is given by

$$
\dot{r}=r^{3} \sin \theta \cos \theta, \quad \dot{\theta}=1 .
$$

Then, by (1.3)

$$
P(\xi, \lambda)=\int_{0}^{2 \pi} d \theta=2 \pi
$$

This finishes the proof.

Proposition 6. System (1.12) has an isochronous center at the origin of period $2 \pi$ and it also has two isochronous centers outside the origin of period $\pi$. 
Proof: This system has centers at the origin and at the points $( \pm 1,0)$. To prove that they are isochronous we consider the complex variable $z=x+\imath y$, which reduces our system to the equation

$$
\dot{z}=\imath z(1+z)(1-z) \text {. }
$$

By direct integration we obtain that the solution $z(t)=z(t, \xi)$ which verifies the initial condition $z(0)=x(0)+\imath y(0)$ with $x(0)=\xi, y(0)=0$ can be computed from relation

$$
z(t)^{2}=\frac{\xi^{2} e^{2 \imath t}}{1+\xi^{2}\left(e^{2 \imath t}-1\right)} .
$$

Then it is clear that the solutions of (2.11) have constant period at a neighborhood of each of its equilibrium points : $z=0,-1,1$. Finally, since for (1.12) the eigenvalues associated to the origin are $\imath,-\imath$ and the ones associated to the points $( \pm 1,0)$ are $\pm 2 \imath, \mp 2 \imath$, the proof is complete.

Proof of Theorem 2 The proof follows from Proposition 3 with $A=1$, Proposition 4 with $A=2$, Proposition 5 and Proposition 6 .

\section{Case II.}

Here we consider general systems of type (1.13) with $\lambda=(a, \eta, \theta, \mu, \nu, \xi, \omega) \neq$ 0 and verifying condition (II) of Theorem 1 . Such systems are symmetric with respect to two orthogonal lines. After a rotation we can assume that the symmetry-axes are the coordinate axes and the systems remain of the type

$$
\begin{array}{ll}
\dot{x}= & -y+a x^{2} y+b y^{3} \\
\dot{y}= & x+c x^{3}+d x y^{2},
\end{array}
$$

with $a, b, c, d$ not all zero.

In order to obtain a more convenient expression for the period coefficients we write (3.1) in the form

$$
\begin{array}{ll}
\dot{x}= & -y+(6 A+B-3 D) x^{2} y+(C-A+D) y^{3} \\
\dot{y}= & x+(C-A-D) x^{3}+(6 A-B+3 D) x y^{2},
\end{array}
$$

with $\lambda=(A, B, C, D) \in \mathbb{R}^{4}-\{0\}$. 
For this system the period coefficients $p_{2 k}(\lambda), k=1,2,3$ under the condition that $p_{j}(\lambda)=0$ for $j<2 k$ are

$$
\begin{aligned}
& p_{2}(\lambda)=-\frac{\pi}{2} B, \\
& p_{4}(\lambda)=\frac{3 \pi}{2}\left(A^{2}-C^{2}-D^{2}\right), \\
& p_{6}(\lambda)=-\frac{3 \pi}{8} C D(A+5 C) .
\end{aligned}
$$

Hence, assuming $p_{2}(\lambda)=p_{4}(\lambda)=0$ we have

$$
p_{8}(\lambda)=\frac{3 \pi}{16} C D\left(-252 A C+36 C^{2}-21 A D-125 C D-54 D^{2}\right) .
$$

Theorem 7. The system (3.2) with $\lambda \neq 0$ has an isochronous center at the origin if and only if $B=0$ and one of the following relations hold:

(a) $C=A-D=0$,

(b) $C=A+D=0$,

(c) $D=A-C=0$,

(d) $D=A+C=0$.

Proof: Conditions $p_{2}(\lambda)=p_{4}(\lambda)=0$ and $A+5 C=0$ ( which makes $p_{6}(\lambda)$ vanish) give $B=0, A=-5 C$ and $D= \pm \sqrt{24} C$. Then the coefficient $p_{8}(\lambda)$ is

$$
p_{8}(\lambda)=-90 C^{4} \text {. }
$$

From this it follows that the conditions in the statement of the theorem are necessary in order to have an isochronous center at the origin.

Now we consider $B=0$. System (3.2) under condition (a) (respectively condition (b)) correspond to system (2.1) of Proposition 3 (respectively system (2.9) of Proposition 4). Moreover, system (3.2) under condition (c) (respectively condition (d)) correspond, modulus a linear change of coordinates to system (1.11) (respectively system (1.12)). This finishes the proof.

Corollary 8. If $M \neq 0$, system

$$
\begin{aligned}
& \dot{x}=-y-3(5 \pm \sqrt{6}) M x^{2} y+(3 \pm \sqrt{6}) M y^{3} \\
& \dot{y}=\quad x+(3 \mp \sqrt{6}) M x^{3}+2(-5 \pm \sqrt{6}) M x y^{2},
\end{aligned}
$$

has a weak center of order 3 at the origin. 
Proof: This system is obtained by letting $B=0, A=-5 C, D= \pm 2 \sqrt{6} C$ and $C=\frac{1}{2} M$ in (3.2). Therefore we have $p_{2}=p_{4}=p_{6}=0$ and $p_{8}=$ $-\frac{45}{8} M^{4}$.

Corollary 9. If the origin for system (3.2) is not an isochronal, then it is a weak center of order at most three.

\section{Cases $\mathbf{H}$ and III.}

In this section we prove that our system (1.13) under condition ( $\mathrm{H})$ or III never has an isochronous center at the origin.

First we consider system (1.13) under condition (H) of Theorem 1. It becomes

$$
\begin{aligned}
(4.1)_{\dot{y}}^{\dot{x}}= & -y-(\omega+\theta) x^{3}-(\eta-3 \mu) x^{2} y-3(\omega-\theta) x y^{2}-(\mu-\nu) y^{3} \\
& x+(\mu+\nu) x^{3}+3(\omega+\theta) x^{2} y+(\eta-3 \mu) x y^{2}+(\omega-\theta) y^{3},
\end{aligned}
$$

with $\lambda=(\eta, \omega, \theta, \mu, \nu) \in \mathbb{R}^{5}$.

The first even period coefficients are

$$
\begin{aligned}
& p_{2}(\lambda)=\frac{\pi}{2} \eta, \\
& p_{4}(\lambda)=-\frac{\pi}{32}\left(-7 \eta^{2}+48 \mu^{2}+24 \mu \nu+48 \nu^{2}+192 \omega^{2}+48 \theta^{2}\right) .
\end{aligned}
$$

From this we obtain

Theorem 10. The origin is a weak center of order zero for system (4.1) with $\lambda \neq 0$ if $\eta \neq 0$ and of order one if $\eta=0$.

On the other hand, system (1.13) under the condition III of Theorem 1 is

$$
\begin{array}{ll}
\dot{x}= & -y-(\theta-a) x^{3}+3 \mu x^{2} y-(2 a-3 \theta) x y^{2}-\mu y^{3} \\
\dot{y}= & x+\mu x^{3}+(3 \theta+2 a) x^{2} y-3 \mu x y^{2}-(\theta+a) y^{3},
\end{array}
$$

with $\lambda=(a, \theta, \mu) \in \mathbb{R}^{3}$ such that $a^{2}=4\left(\theta^{2}+\mu^{2}\right)$.

The period coefficient $p_{2}(\lambda)$ always vanishes and

$$
p_{4}(\lambda)=-\frac{\pi}{4}\left(-a^{2}+6 \mu^{2}+6 \theta^{2}\right) .
$$

Under the condition $a^{2}=4\left(\theta^{2}+\mu^{2}\right)$ the coefficient $p_{4}$ takes the form

$$
p_{4}(\lambda)=-\frac{\pi}{8} a^{2} .
$$

Then we have the following result : 
Theorem 11. If $\lambda \neq 0$, the origin is a weak center of order one for system (4.2).

\section{Isochronous outside the origin}

Theorem 12. Let

$$
\begin{array}{ll}
\dot{x}= & -y+p_{3}(x, y) \\
\dot{y}= & x+q_{3}(x, y)
\end{array} .
$$

Such system has an isochronous center at $\left(x_{0}, y_{0}\right) \neq(0,0)$ if and only if it can be reduced to one of the systems (1.9), (1.12), given in Section 1, through a linear change of coordinates.

Proof: Suppose $p_{3}$ and $q_{3}$ are written as follows :

$$
\begin{aligned}
& \mathrm{p}_{3}(\mathrm{x}, \mathrm{y})=(\mathrm{a}-\omega-\theta) \mathrm{x}^{3}-(\eta-3 \mu) \mathrm{x}^{2} \mathrm{y}-(3 \omega-3 \theta+2 \mathrm{a}-\xi) \mathrm{xy}^{2}-(\mu-\nu) \mathrm{y}^{3} \\
& \mathrm{q}_{3}(\mathrm{x}, \mathrm{y})=(\mu+\nu) \mathrm{x}^{3}+(3 \omega+3 \theta+2 \mathrm{a}) \mathrm{x}^{2} \mathrm{y}+(\eta-3 \mu+\mathrm{b}) \mathrm{xy}^{2}+(\omega-\theta-\mathrm{a}) \mathrm{y}^{3}
\end{aligned}
$$

Since this normal form is invariant by rotations and homothetic linear transformations, we assume that $\left(x_{0}, y_{0}\right)=(1,0)$. Therefore

$$
\omega+\theta-a=0 \text { and } \mu+\nu=-1 .
$$

Moreover, since the eigenvalues of system at $(1,0)$ must have vanishing real part, we have also $\omega+\theta=0$ (which implies $a=0$ ) and $3 \mu-\eta-1>0$.

Then we obtain

$$
\begin{array}{ll}
\dot{x}= & -y-(\eta-3 \mu) x^{2} y-(6 \omega-\xi) x y^{2}-(1+2 \mu) y^{3} \\
\dot{y}= & x-x^{3}+(\eta-3 \mu+b) x y^{2}+2 \omega y^{3} .
\end{array}
$$

Performing a translation that moves the point $(1,0)$ to the origin, plus the transformation $(x, y) \rightarrow(\lambda x, y)$, with $\lambda=\sqrt{\frac{3 \mu-\eta-1}{2}}$, and also reescaling we obtain

$$
\begin{aligned}
\dot{\mathrm{x}}=\mathrm{y}[-1 & +2 \lambda(\eta-3 \mu) \mathrm{x}+(6 \omega-\xi) \mathrm{y}-2 \lambda^{4}(\eta-3 \mu) \mathrm{x}^{2}+2 \lambda^{3}(6 \omega-\xi) \mathrm{xy} \\
& \left.+2 \lambda^{2}(1+2 \mu) \mathrm{y}^{2}\right] \\
\dot{\mathrm{y}}= & \mathrm{x}+3 \lambda^{3} \mathrm{x}^{2}-\lambda(\eta-3 \mu+\mathrm{b}) \mathrm{y}^{2}+2 \lambda^{6} \mathrm{x}^{3}-2 \lambda^{4}(\eta-3 \mu+\mathrm{b}) \mathrm{xy}{ }^{2} \\
& -4 \lambda^{3} \omega \mathrm{y}^{3} .
\end{aligned}
$$

The first Liapunov quantity is

$$
\eta_{2}=\frac{\lambda}{8}[2(\xi-6 \omega) b-\xi(3 \mu-\eta-1)]
$$


Case A. Here we assume $\xi-6 \omega \neq 0$. We will prove that in this case, when the origin is a center for the system (5.4), it is a weak center of order 0 .

To vanish $\eta_{2}$, we must have

$$
b=\frac{\xi(3 \mu-\eta-1)}{2(\xi-6 \omega)}
$$

and the next Liapunov quantity is

$$
\eta_{3}=\frac{\xi \lambda(3 \mu-\eta-1)^{2}}{96(6 \omega-\xi)}[-\xi(11-9 \eta+47 \mu)+24 \omega(2-3 \eta+14 \mu)]
$$

First we consider the subcase $11-9 \eta+47 \mu \neq 0$. Then

$$
\xi=24 \omega \frac{2-3 \eta+14 \mu}{11-9 \eta+47 \mu}
$$

and the Liapunov quantity

$$
\eta_{4}=\frac{\lambda \omega(2-3 \eta+14 \mu)(3 \mu-\eta-1)^{4}(1+\mu)}{6(11-9 \eta+47 \mu)^{3}}\left[(11-9 \eta+47 \mu)^{2}+3780 \omega^{2}\right]
$$

If $2-3 \eta+14 \mu=0$, we have $\xi=0$ and $b=0$ (and the system is Hamiltonian). In this case $3 \mu-\eta-1=-\frac{5}{3}(\mu+1)$ and the first period coefficient is

$$
p_{2}=\frac{10}{9}\left[324 \omega^{2}-(1+\mu)^{2}(67+100 \mu)\right]>0
$$

When $2-3 \eta+14 \mu \neq 0, \eta_{4}=0$ if and only if $\mu=-1$. Then $3 \mu-\eta-1=$ $-(\eta+4)$ and

$$
p_{2}=2\left[-(\eta+4)^{3}+3(\eta+4)^{2}+20 \omega^{2}\right]>0 .
$$

Now we consider the subcase $11-9 \eta+47 \mu=0$. Then $\eta_{3}=0$ if and only if $\omega=0$ or $2-3 \eta+14 \mu=0$. If $\omega=0$, we have $b=-\frac{10}{9}(\mu+1)$ and $3 \mu-\eta-1=-20(\mu+1)$. But

$$
\eta_{4}=-\frac{250 \sqrt{5}}{177147 \sqrt{2}} \xi(-1-\mu)^{\frac{7}{2}}\left[80(1+\mu)^{2}+189 \xi^{2}\right]
$$

Therefore $\omega \neq 0$ and $2-3 \eta+14 \mu=0$. But in this case, we must have $\mu=-1$ and $\eta=-4$ which imply $3 \mu-\eta-1=0$. These finishes Case A). 
Case B. Here we assume $\xi=6 \omega$. Then the condition $\eta_{2}=0$ implies $\xi=\omega=0$. System (??) remains symmetric with respect to axes $x$ and $y$, and therefore, the origin is a center for system (5.4). The first period coefficients are of the form

$$
p_{k}=\pi(3 \mu-\eta-1)^{k} q_{k}(\mu, \eta, b) \quad k=2,4,6,8 .
$$

with $q_{k}$ a polynomial of degree $k$ at $\mu, \eta$ and $b$. By consider $q_{2}, \cdots, q_{8}$ as polynomials in $\mu$ we call $R_{2, k}$ the resultant of $q_{2}$ and $q_{k}$ for $k=4,6,8$. Then

$$
R_{2, k}=\left(24+6 \eta-9 b+2 b^{2}\right) r_{k-1}(\eta, b)
$$

with $r_{k-1}(\eta, b)$ a polynomial which is of degree $k-1$ in $\eta$.

If we put $\eta=-\frac{1}{6}\left(24-9 b+2 b^{2}\right)$ in the polynomials $q_{k}$ for $k=4,6,8$, we obtain

$$
q_{k}=\left(18+18 \mu-9 b+2 b^{2}\right) Q_{k}(\mu, b)
$$

with $Q_{2}(\mu, b)=\frac{1}{6}\left(102+72 \mu-51 b+8 b^{2}\right)$.

Since the condition $18+18 \mu-9 b+2 b^{2}=0$ implies $3 \mu-\eta-1=0$ we must study the case

$$
\mu=-\frac{1}{72}\left(102-51 b+8 b^{2}\right) .
$$

In this case, $Q_{4}$ is a cubic polynomial in $b$ which have no common root with the polynomials $Q_{6}$ and $Q_{8}$.

Therefore we assume $24+6 \eta-9 b+2 b^{2} \neq 0$. Now we consider the polynomials $r_{k-1}(\eta, b), k=4,6,8$ as polynomials in $\eta$ and compute the resultants $R_{2,4, k}$ between $r_{3}$ and $r_{k-1}$ for $k=6,8$. We obtain

$$
\begin{aligned}
& R_{2,4,6}=b^{4}(b-1)(b-6)^{5} m_{13}(b) \quad \text { and } \\
& R_{2,4,8}=b^{6}(b-1)(b-6)^{5} m_{19}(b)
\end{aligned}
$$

Since the polynomials $m_{13}$ and $m_{19}$ have no common roots, we must analyse the cases $b=0, b=1$ and $b=6$.

Case $b=0$. In this case we obtain

$$
\begin{aligned}
R_{2,4}= & c_{1}(4+\eta)^{2}\left(2250+1590 \eta+289 \eta^{2}\right) \\
R_{2,6}= & c_{2}(4+\eta)^{3}\left(132975+90585 \eta+16308 \eta^{2}+242 \eta^{3}\right) \\
R_{2,8}= & c_{3}(4+\eta)^{4}\left(611448750+811069200 \eta+403332894 \eta^{2}+89213748 \eta^{3}\right. \\
& \left.+7414729 \eta^{4}\right)
\end{aligned}
$$


with $c_{1}, c_{2}, c_{3}$ non vanishing constants. Then $R_{2,4}=0$ if and only if $\eta=-4$. With this value of $\eta$ we have $3 \mu-\eta-1=3(\mu+1)$ and

$$
q_{2}=18(1+\mu)(17+12 \mu) \text {. }
$$

Therefore in this case the origin is a weak center of order 0 of system (5.4). Case $b=1$. In this case we obtain

$$
\begin{aligned}
R_{2,4}= & c_{1}(17+6 \eta)^{2}\left(128203+81342 \eta+13872 \eta^{2}\right), \\
R_{2,6}= & c_{2}(17+6 \eta)^{2}\left(59808166387+61367463684 \eta+21342210876 \eta^{2}\right. \\
& \left.+2547210240 \eta^{3}+2509056 \eta^{4}\right) \\
R_{2,8}= & c_{3}(17+6 \eta)^{2}(34978219250276851+53432161728297080 \eta \\
& +32138822066897944 \eta^{2}+9489481112169312 \eta^{3} \\
& \left.+1381403003367792 \eta^{4}+85977730831104 \eta^{5}+1845021846528 \eta^{6}\right)
\end{aligned}
$$

with $c_{1}, c_{2}, c_{3}$ non vanishing constants. Then $R_{2,4}=0$ if and only if $\eta=-\frac{17}{6}$. With this value of $\eta$ we have $3 \mu-\eta-1=\frac{1}{6}(18 \mu+11)$ and

$$
q_{2}=\frac{1}{6}(11+8 \mu)(59+72 \mu) \text {. }
$$

Therefore in this case the origin is also a weak center of order 0 of system (5.4).

Case $b=6$. In this case we obtain

$$
\begin{aligned}
R_{2,4}= & c_{1}(3+\eta)^{2}(7+\eta)(523+289 \eta) \\
R_{2,6}= & c_{2}(3+\eta)^{2}(7+\eta)(543850779+323868874 \eta \\
& \left.+11136599 \eta^{2}+484 \eta^{3}\right) \\
R_{2,8}= & c_{3}(3+\eta)^{2}(7+\eta)(957359796516423+605631187512333 \eta \\
& +40017853636902 \eta^{2}+1191561383482 \eta^{3}+5683972739 \eta^{4} \\
& \left.+7414729 \eta^{5}\right)
\end{aligned}
$$

with $c_{1}, c_{2}, c_{3}$ non vanishing constants.

If $\eta=-\frac{523}{289}$ we have $R_{2,6} \neq 0,3 \mu-\eta-1=\frac{3}{289}(289 \mu+78)$ and

$$
\begin{aligned}
q_{2}= & \frac{36}{83521}(43+1734 \mu)(-92+289 \mu) \\
q_{4}= & \frac{1296}{6975757441}(-92+289 \mu)(638989804+21531733741 \mu \\
& \left.+24374267514 \mu^{2}+20854859616 * \mu^{3}\right)
\end{aligned}
$$

Therefore system (5.4) have a weak center of order at most 1 at the origin. 
For $\eta=-7$, we have $3 \mu-\eta-1=3(\mu+2)$ and

$$
q_{2}=36(2+\mu)(7+6 \mu) \text {. }
$$

Hence, in this case the origin is a weak center of order 0 of system (5.4).

Finally, when $\eta=-3$, we have $3 \mu-\eta-1=3 \mu+2$ and

$$
q_{2}=108 \mu(1+2 \mu) \text {. }
$$

But system (5.3) under conditions: $\omega=\xi=0, b=6, \eta=-3$ and $\mu=-\frac{1}{2}$ (respectively, $\mu=0$ ), is exactly system (1.9) (respectively system (1.12)). Therefore, only in these two cases we have an isochronous center outside the origin.

The following Corollary follows from the above proof.

Corollary 13. A non isochronous center different from the origin of system (5.1) is a weak center of order at most one.

\section{References}

[1] C. Chicone and M. Jacobs, Bifurcations of critical periods for plane vector fields, Trans. Amer. Math. Soc. 312, No. 2, pp. 433-486, (1989).

[2] W. S. Loud, Behavior of the period of solutions of certain plane autonomous systems near centers, Contributions to Differential Equations 3 , pp. $(21-36),(1964)$.

[3] N. A. Lukashevich, isochronicity of center for certain systems of differential equations, Differential Equations 1, pp. 220 - 226, (1965).

[4] K. E. Malkin, Criteria for center of a differential equation, Volg. Matem. Sbornik 2, pp. $87-91,(1964)$.

[5] I. I. Pleshkan, A new method of investigating the isochronicity of a system of two differential equations, Differential Equations 5, pp. $796-$ $802,(1969)$.

[6] C. Rousseau and D. Schlomiuk, Cubic vector fields symmetric with respect to a center, preprint, Université de Montréal, (1992). 
[7] C. K. Siegel and J. K. Moser, Lectures on celestial mechanics, SpringerVerlag, New York, (1971).

[8] K. S. Sibirsky, On the number of limit cycles in the neighborhood of a singular point, Differential Equations 1, pp. $36-47,(1965)$.

[9] M. Urabe, Potential forces which yield periodic motions of a fixed period, J. Math. Mech. 10, pp. 569 - 578, (1961).

Received : July 05, 1995.

\section{Víctor Guíñez}

Departamento de Matemáticas y Cs. de la Computación

Universidad de Santiago de Chile

Casilla 307

Correo 2

Santiago - Chile

\section{Jaime Figueroa}

\section{Eduardo Sáez}

Departamento de Matemáticas

Universidad Técnica Federico Santa María

Casilla 110-V

Valparaíso - Chile 